\title{
A Real-Time Information System Application of Industrial Training Using Android Apps and GSM Module
}

\author{
Devi Handaya ${ }^{1}$, Pranoto Budi Laksono ${ }^{2}$ \\ \{devi.handaya.el@gmail.com ${ }^{1}$, pranoto@poltek-gt.ac.id ${ }^{2}$ \} \\ Electronics Engineering, Politeknik PGRI Banten, Cilegon, Indonesia ${ }^{1}$, Electronics Engineering, \\ Politeknik Gajah Tunggal, Tangerang, Indonesia ${ }^{2}$
}

\begin{abstract}
This research builds a real time information system application for industry training. The system is built to support employee competency improvement through training schedule notification, attendance confirmation, monitoring of pre-test and posttest results. The design of the application is build using android studio, while the hardware using Arduino mega2560 and GSM module. SMS notification system transmitted by GSM module, utilizing AT Command. Experiments were conducted by testing the conformity of the results of notification delivery via SMS, confirmation of participation or attendance of training, monitoring of pre-test and post-test results on mobile phones. Sophistication is based on data contained in the sending system or online database and the receiving system or mobile phone. Based on the experiment, it was found that the system can deliver SMS notification according to schedule. Confirmation of attendance, monitoring of pre-test and post-test results conducted by trainee has been in accordance with online databases.
\end{abstract}

Keywords: real-time information system, industrial training, android apps, GSM module.

\section{Introduction}

The era of digital information is one form of technology that develops even in the industrial world as a supporter of activities in terms of assisting Human Resources (HR) management. Form of HR improvement such as through employee training activities. In the HR management literature, HR training is defined as the application of the official process to provide knowledge and assist employees in obtaining competency needs so as to gain job satisfaction [1].

In terms of training activities management, training providers manage data only on an internal scope so that employees do not have easy access to know their competencies and schedule. Thus, a system is needed that allows employees to be able to access information and schedule notices of training. Any employee who is lagging behind the training information can not follow the training activity and the employee's competence is not achieved. HR training is a systematic process in terms of increasing employee competency [2]. If the competence of employees is not achieved, then the goal of the company that hopes to increase productivity of its products will be hampered.

ICCSET 2018, October 25-26, Kudus, Indonesia

Copyright (C) 2018 EAI

DOI 10.4108/eai.24-10-2018.2280541 
The design of training information system of industrial employee competence is still minimal conducted by the researcher, hence the researcher try to find its approach, that is on HR management. Information systems research can solve many practical problems such as HR management efficiency, simple, and easy to apply [3]. The system is also applied in conducting employee performance appraisals for promotion [4].

Information systems designed on android apps have also been made to provide productivity information on injection molding machines and paperless. This system uses the database so it can be monitored in real time [5]. Real time information is also performed on monitoring lithium-ion batteries in Electric Vehicles (EV). The Information transmitted via WIFI communication to mobile phone [6]. The information system using the SMS module is used on flood water level monitoring. Utilizing Arduino and GSM module by delivering SMS notification to the public [7]. It is also similar to measuring the efficiency of solar, moisture, and light panels informed by SMS using the Arduino mega 2560 and GSM modules [8], dan Arduino Uno and GSM modules for radiation monitoring [9].

Based on the needs in solving problems and technological developments, the researcher undertook the project in the form of support to information systems of employee competence improvement through training schedule notification. Researchers also designed information systems by utilizing online databases, so employees can access in real time using android smartphone. Thus, conducted research related to real time information system for industrial training on android smartphone.

\section{Research Method and Design of Training Information System}

This research has two main steps. At the software design step, the system is built using android programming for mobile applications. At the hardware design step, the system uses an Arduino microcontroller that is connected to the Global System for Mobile Communication (GSM) module facility for notification delivery. The database source utilizes the online Data Base Management System (DBMS). Here the block diagram of Information System.

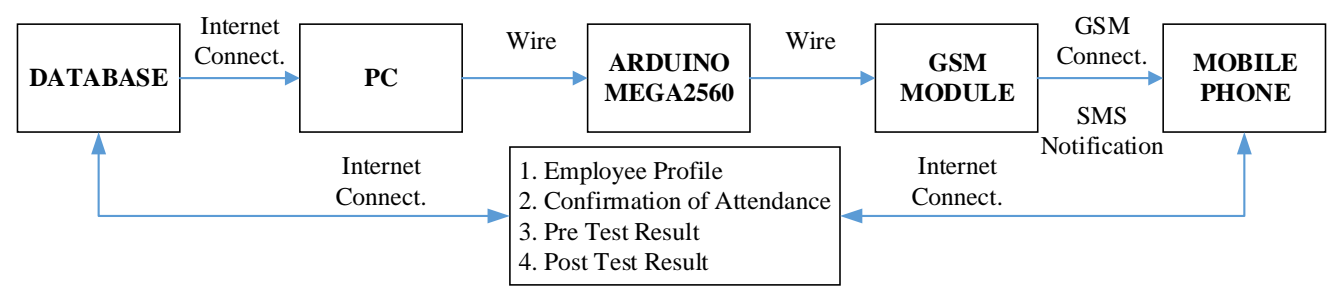

Fig. 1. Block diagram of information system for industrial training.

The database delivers the information to the Personal Computer (PC) about the employee who will attend the training. The PC will instruct mega 2560 and GSM module to deliver an industrial training notification SMS and ask for confirmation of its presence. Furthermore, trainees will confirm presence through the Android apps. In addition, the application will also provide information related to employee profile and test results in its participation. 
The information system designed is a system that can deliver information both for trainee and training providers. With a view to being able to provide information, the design of this research is considered to be able to provide information since the training activities will begin until ends. The design results will be tested on the mobile phone. The following model block diagram of the application and testing on the mobile phone.

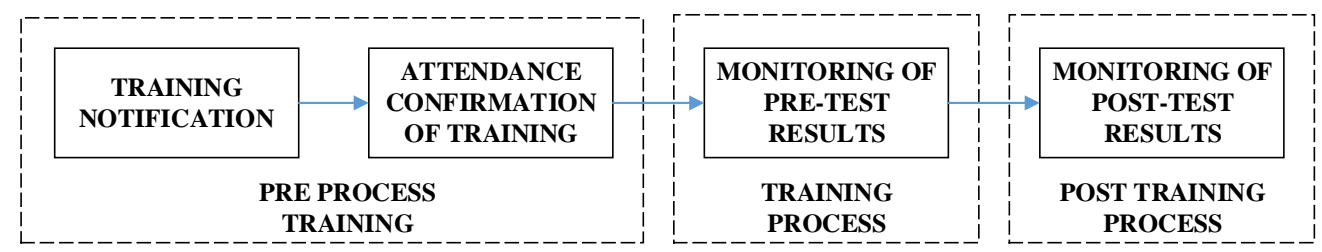

Fig. 2. Block diagram of implementation model and testing on mobile phone.

Testing in the pre process training step of the suitability of delivery notification results via SMS and confirmation of participation / attendance training. In the test when the training process in the form of access data employee / trainee and monitor of pre-test results. After the training, it can monitor post-test results during the training.

\subsection{Design of Training Information System on Android Apps}

Android application designed in this study using android studio software. The design on android apps can be seen in Fig. 3. Before going to the main feature, login required using NIK and password. The main features of android apps are as follows.

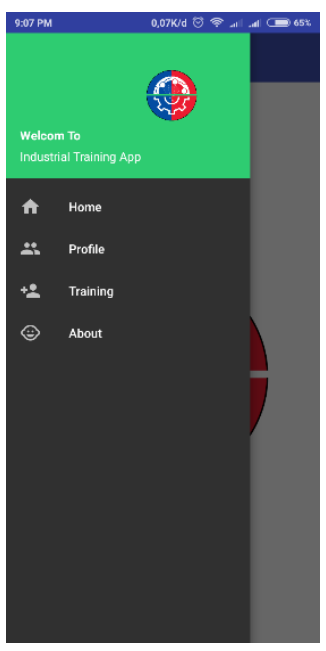

(a)

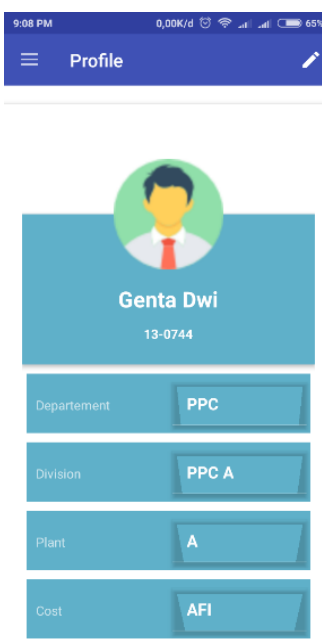

(b)

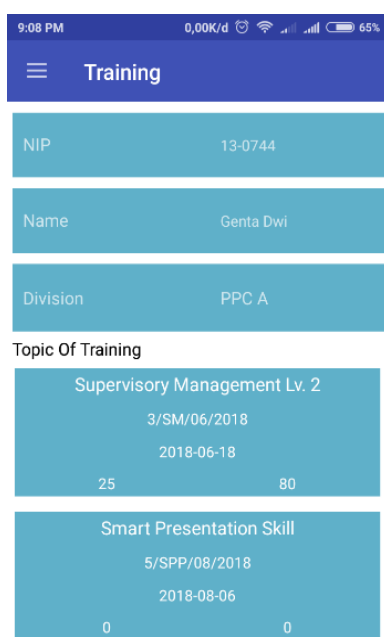

(c)

Fig. 3. The view of industrial training information systems on android apps : (a) The main view of information systems, (b) Display on employee profile feature, and (c) The main view on the training list. 
The feature displays of the employees profile who have been registered in $\mathrm{HR}$ Departement. The feature include employee name, Employer Identity Number/NIK, department, division, plant, cost, grade, position, status employee, group, employee type, sex, and date of entry. The training list is a feature provided to display the training topics to be followed by the employee. In this feature, the employee can confirm the attendance of the training and see the pre-test and the test post results during the training.

\subsection{Design of Hardware for Training Information System}

In this research using microcontroller mega 2560 as processor of message sender. Information delivered via SMS intends to be a good reminder to employees who own a smartphone or not. The microcontroller connected with the Sim8001 GSM module is programmed to deliver the information. GSM module is compatible with Arduino by utilizing AT Command protocol. AT Command is a set of commands as a form of data communication on GSM communication module [10]. The AT Command protocol is part of the development of IoT [11]. ATCommand used in this study is AT+CMGF which serves to determine the mode format of the terminal. The text format mode uses a value of 1 . AT+CMGS serves as a format for sending messages.

\section{Result and Discussion}

\subsection{Test of SMS notifications}

In this research, system flow begins with the delivery of information of industrial training in the form of SMS notification to the trainee. The result of testing the suitability of industrial training notification to trainee number can be seen in the following figure.

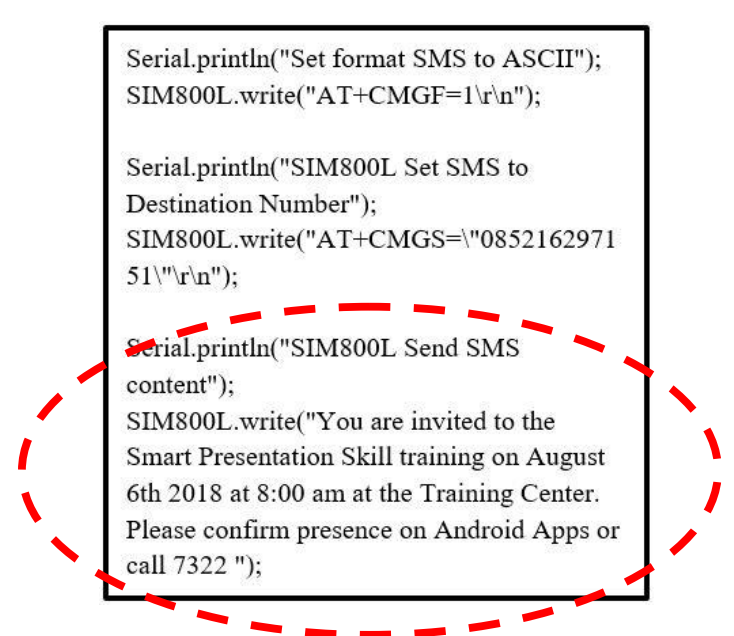

(a)

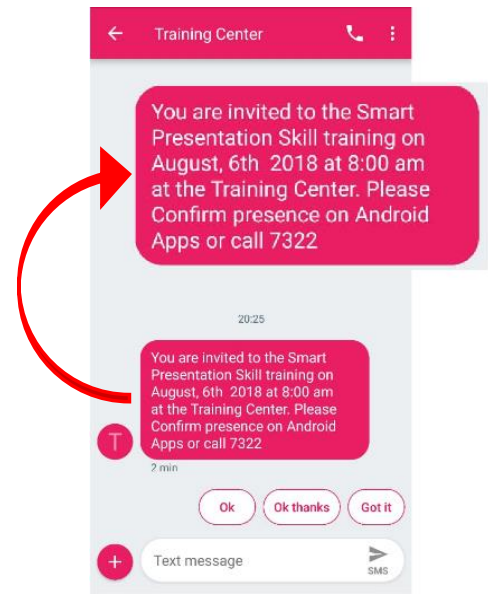

(b)

Fig. 4. (a) Screenshot of SMS notification sender system and (b) Screenshot in receiving system. 
Based on Fig.4, it can be seen that messages are delivered appropriately. The contents of messages delivered through the system can be well received by trainee so that employees who will undergo the training has been given information by training providers and is expected to meet the invitation by doing confirmation as directed on the message. Confirmation can be made either by contacting the training provider directly, or through android app.

\subsection{Test of employee / trainee data access}

In testing employee/trainee data access done by considering the suitability of employee data contained on android apss with online database. Here is a picture of the test results of employee data access in Fig. 5.

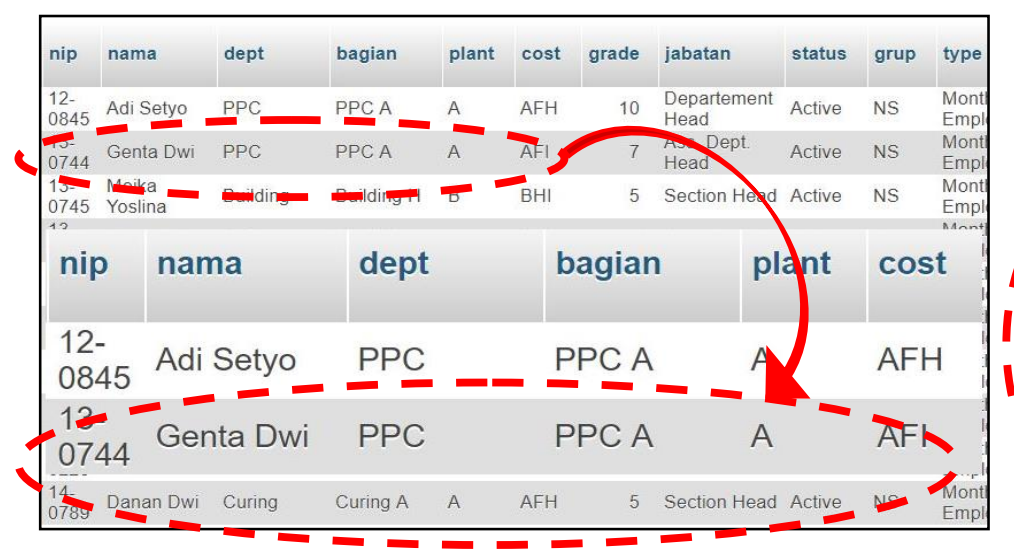

(a)

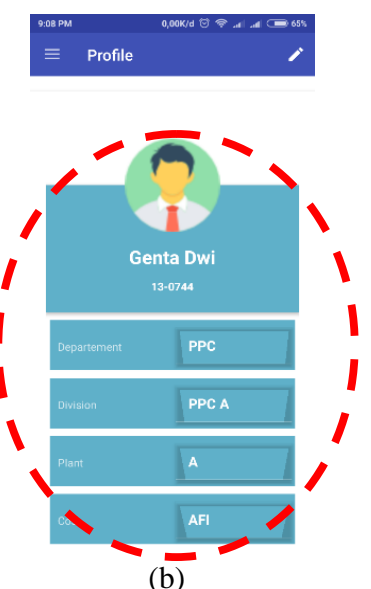

(b)

Fig. 5. Test of employee / trainee data access : (a) Display on employee profile data in android apps and (b) Screenshot in online database.

Based on the tests performed on the employee data access, it can be seen that the existing employee data on android apps is in accordance with the data that the system has on the online database. This can be proved in Fig. 5 which both contain the same value. Thus, participants can easily see the appropriateness between the actual employee data and the data that the provider has. Employee profile inaccuracies can lead to some errors such as training topics and training certificates that employees will receive.

\subsection{Test on confirmation of training participation / attendance}

In this test, the employee / trainee confirms attendance on the android apps. The results of the confirmation of attendance can be seen in the following figure. 


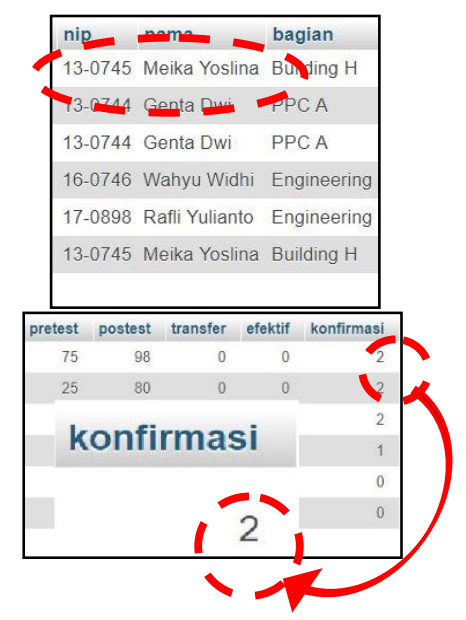

(a)

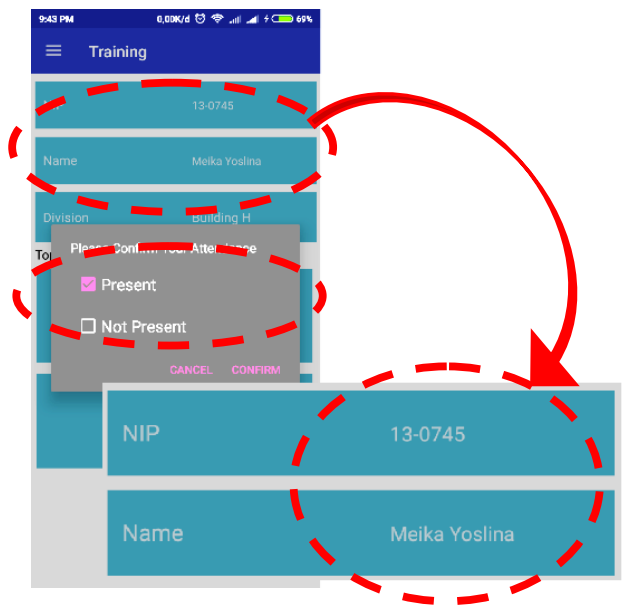

(b)

Fig. 6. Test on confirmation of training participation : (a) Display on confirmation of attendance in android apps and (b) Screenshot in online database.

The results shown in the figure above show that android apps are able to provide attendance confirmation to the system shown in the online database. For confirmation is attend, then the database will bring up the value 2, while for confirmation is not attend, then the database will bring up the value 1 . Value 0 indicates that employees have not confirmed through android apps.

With the presence of confirmation features on android apps, participants are given the convenience to make quick confirmation without the need to contact the provider directly. In addition, the confirmation process is also easy to do, which can be done anywhere and anytime. Benefits received by the training provider, which can monitor the attendance of participants in the training to be implemented. So the provider can prepare various matters related to location capacity, consumption, room comfort, loudspeakers, and so on.

\subsection{Tests on monitoring the results of pre-test and post-test during training}

In the following tests, employees / trainee who have attended the training, can monitor the results of the training. The results are obtained from a comparison of pre-test and post-test values. Here is the picture of the pre-test and post-test value of the trainees. 


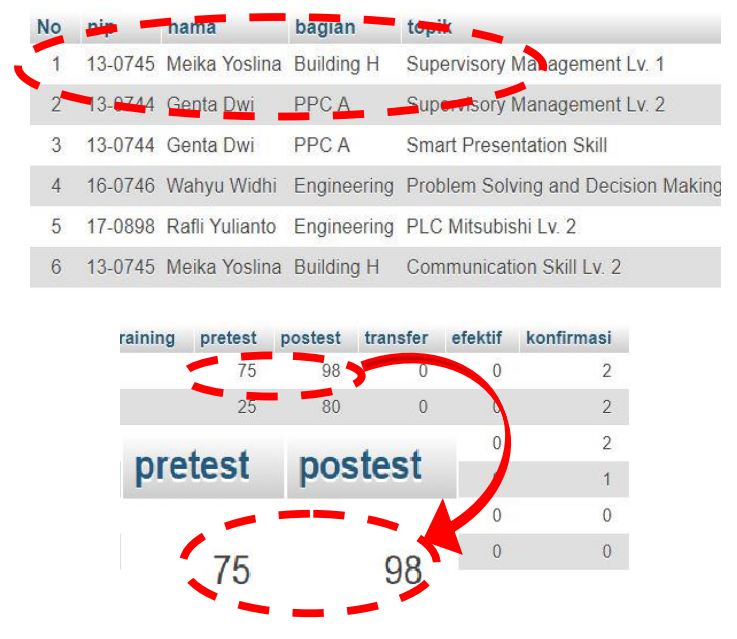

(a)

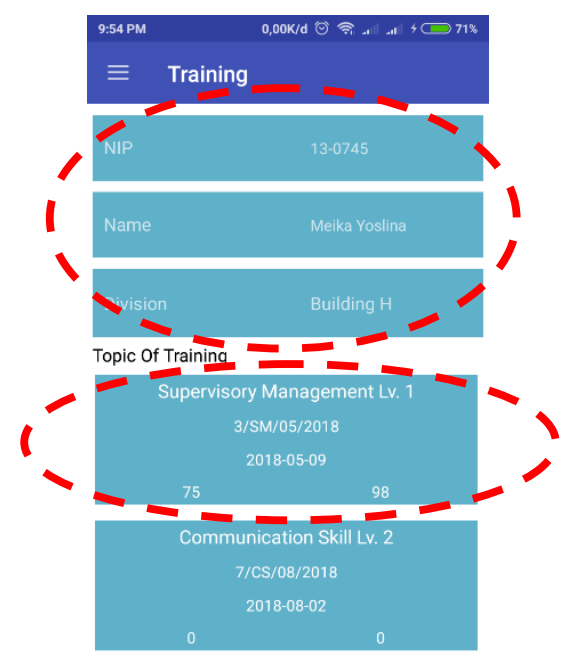

(b)

Fig. 7. Tests on monitoring the results of pre-test and post-test during training : (a) Display of pre-test and post-test results on android apps. and (b) Display on online database.

The results shown in the picture above prove that the data entered in the online database is also displayed on the android apps. The pre-test value can be monitored after several hours of pre-test. This is used as a guide for trainee and training providers. For new post-test values will appear after the trainee do the training. Pre-test and post-test comparison values serve as a benchmark for the success of trainee and provider in industrial training activities. The results of these values are used as a form of competency achievement in achieving a competency.

\section{Conclusion}

Real time information systems on android applications are successfully designed with the aim of increasing employee participation in industrial training. SMS notifications transmitted by GSM module successfully deliver all information to employees. So it is expected that employees can arrange their schedule to attend industrial training activities. The condition of the data between the online database with the android app has been appropriate. This is indicated by the employee profile data contained in the android apps has been in accordance with the database. Then the results of employee confirmations on android apps also appear as a value on the online database. A value of 0 for the unconfirmed participant, value 2 for the confirmed participant, and the value of 1 for the confirmed participant is not attend. With the presence of the confirmation menu, the training developer can adjust the location capacity, consumption, room comfort, loudspeakers, and so forth. The value of pre-test and post-test results that appear on android apps also matches the online database. This helps the participants and the training developer in knowing the benchmarks of competence achievement as an evaluation material for further industrial training. 


\section{Acknowledgments}

This research was supported by Directorate of Research and Coomunity Service - Directorate General of Research and Development Strengthening - Ministry of Research, Technology, and Higher Education of Republic Indonesia which provides funding and opportunity to researchers so as to carry out this research .

\section{References}

[1] M. Armstrong, Armstrong's Handbook of Human Resource Management Practic. London: Kogan Page, 2009.

[2] G. Latham, Human Resource Training and Development. Annual Review of Psychology. 1988.

[3] G. Ying and G. Xiao-hui, "J2EE-Based Human Resources's Management Information System Design and Implementation," in 2nd IEEE Intl. Conference on Computer Science and Information Technology, 2009, pp. 25-28.

[4] D. M. Agustina, M. J. D. Sunarto, and K. Jatmika, "Sistem Informasi Penilaian Kinerja Pegawai Pada Badan Kepegawaian dan Diklat Surabaya," Sist. Inf., vol. 3, no. 2, pp. 72-77, 2014.

[5] M. Rubenstein, D. Handaya, and W. Slamet, "Design and Implementation of Productivity Monitoring System in Rubber Injection Molding Machine in Real Time Based on Visual Studio and Android Application," in IOP Conference Series: Materials Science and Engineering, 2018.

[6] W. Menghua and X. Bing, "A Real-Time Android-Based Monitoring System for the Power Lithium-Ion Battery Used on EVs," in Proceedings - 10th International Conference on Intelligent Computation Technology and Automation, ICICTA 2017, 2017, pp. 245-249.

[7] A. N. Yumang et al., "Real-Time Flood Water Level Monitoring System with SMS Notification," in HNICEM 2017 - 9th International Conference on Humanoid, Nanotechnology, Information Technology, Communication and Control, Environment and Management, 2018.

[8] Adilah, Nadzlin, and Mahadi, "Development of Solar Efficiency Monitoring System by Using GSM Technology," in Intl. Conference on Space Science and Comm., 2015, pp. 362-365.

[9] N. A. Abd Rahman et al., "GSM Module for Wireless Radiation Monitoring System via SMS," in IOP Conference Series: Materials Science and Engineering, 2018, vol. 298, no. 1.

[10] C. Peijiang and J. Xuehua, "Design and Implementation of Remote Monitoring System Based on GSM,” 2008 IEEE Pacific-Asia Work. Comput. Intell. Ind. Appl., pp. 678-681, 2008.

[11] Z. Zhang, H. Ochiai, and H. Esaki, "An IoT Application-Layer Protocol Modem: A Case Study on Interfacing IEEE 1888 with at Commands," in Proceedings - 2017 Intl. Conf. on Cyber-Enabled Distributed Computing and Knowledge Discovery, CyberC 2017, 2018, pp. 346-349. 